\title{
Adaptación a la población española de la Escala de Perfil de Riesgo de Consumo de Drogas (EPRCD) y estudio de sus propiedades psicométricas
}

\section{Adaptation to the Spanish population of the Substance Use Risk Profile Scale (SURPS) and psychometric properties}

\author{
Fermín Fernández-Calderón*, Carmen Díaz-Batanero*, Antonio J. Rojas-Tejada**, Natalie \\ Castellanos-Ryan***, Óscar M. Lozano-Rojas*. \\ * Departamento de Psicología Clínica y Experimental, Universidad de Huelva. Campus de El Carmen. Avenida de las Fuerzas \\ Armadas, s/n. 21071, Huelva, España; ** Departamento de Psicología, Universidad de Almería, La Cañada de San Urbano, \\ 04120 Almería, España; *** Universidad de Montreal, Canadá.
}

\section{Resumen}

La identificación de diferentes perfiles de personalidad de riesgo para el consumo problemático de drogas es útil para prevenir problemas relacionados con las drogas. Este estudio tiene como objetivo analizar las propiedades psicométricas de una nueva versión de la Substance Use Risk Profile Scale (SURPS) en estudiantes universitarios españoles. Estudio de diseño transversal en el que participaron 455 estudiantes de cuatro universidades españolas. La nueva versión de la SURPS adaptada a la población española fue administrada junto a la Escala de Desesperanza de Beck, la UPPS-P, el inventario de ansiedadestado (STAI) y el test AUDIT. La consistencia interna de las cuatro subescalas de la SURPS osciló entre 0,652 y 0,806. Los coeficientes de fiabilidad por el procedimiento de dos mitades oscilaron entre $0,686 \mathrm{y}$ 0,829. La estimación test-retest osciló entre 0,733 y 0,868 . Se replicó la estructura factorial esperada de cuatro dimensiones. Como evidencias de validez convergente, se encontró que las subescalas de la SURPS se relacionaron significativamente con las medidas teóricamente esperadas de otras escalas de personalidad y con el consumo de alcohol. Esta versión de la SURPS constituye un instrumento útil para la medición de rasgos de personalidad relacionados con la vulnerabilidad al consumo de drogas y sus problemas relacionados, pudiendo ser utilizada para estrategias de prevención del consumo de drogas.

Palabras clave: Substance Use Risk Profile Scale; versión española; propiedades psicométricas; personalidad.

\begin{abstract}
The identification of different personality risk profiles for substance misuse is useful in preventing substance-related problems. This study aims to test the psychometric properties of a new version of the Substance Use Risk Profile Scale (SURPS) for Spanish college students. Cross-sectional study with 455 undergraduate students from four Spanish universities. A new version of the SURPS, adapted to the Spanish population, was administered with the Beck Hopelessness Scale, the UPPS-P Impulsive Behavior Scale, the State-Trait Anxiety Inventory (STAI) and the Alcohol Use Disorders Identification Test (AUDIT). Internal consistency reliability ranged between 0.652 and 0.806 for the four SURPS subscales, while reliability estimated by split-half coefficients varied from 0.686 to 0.829 . The estimated test-retest reliability ranged between 0.733 and 0.868 . The expected four-factor structure of the original scale was replicated. As evidence of convergent validity, we found that the SURPS subscales were significantly associated with other conceptually-relevant personality scales and significantly associated with alcohol use measures in theoretically-expected ways. This SURPS version may be a useful instrument for measuring personality traits related to vulnerability to substance use and misuse when targeting personality with preventive interventions.
\end{abstract}

Keywords: Substance Use Risk Profile Scale; Spanish version; psychometric properties; personality. 


\section{Introducción}

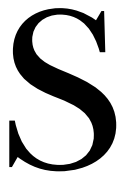

e ha demostrado una fuerte asociación entre rasgos de personalidad, como la impulsividad y la búsqueda de sensaciones, con el consumo de sustancias (Mitchell y Potenza, 2014) y los trastornos por consumo de sustancias (Anderson, Tapert, Moabad, Crowley, y Brown, 2007; Ávila et al., 2016; Hicks, Durbin, Blonigen, Iacono, y McGue, 2012; Kotov, Gamez, Schmidt, y Watson 2010; Sher, Bartholow, y Wood, 2000). Además, los rasgos de personalidad evaluados durante la adolescencia y la adultez temprana han mostrado ser buenos predictores del inicio en el consumo de drogas y la transición a problemas relacionados con dicho consumo (Cho et al., 2015; Gonzálvez, Espada, Guillén-Riquelme, Secades y Orgilés, 2016; Nees et al., 2012).

Para prevenir el abuso de sustancias, es útil contar con instrumentos adecuados que permitan identificar diferentes perfiles de personalidad de riesgo para el consumo problemático de drogas. La Substance Use Risk Profile Scale (SURPS) (Woicik, Stewart, Pihl, y Conrod, 2009) identifica cuatro rasgos de personalidad que han mostrado relación con la vulnerabilidad al consumo de drogas (Castellanos-Ryan y Conrod, 2012): desesperanza (H), sensibilidad a la ansiedad (AS), impulsividad (IMP), y búsqueda de sensaciones (SS). Como señalaron Malmberg et al. (2010), H y AS son rasgos asociados con los procesos de reforzamiento negativo del consumo de sustancias (p. ej., sustancias que alivian estados afectivos negativos), mientras que SS e IMP se asocian con los efectos de refuerzo positivo de las drogas (p. ej., los efectos hedonistas de las sustancias). Desde una perspectiva de prevención selectiva, numerosos estudios ha mostrado la efectividad de las intervenciones centradas en la personalidad para la prevención del abuso de sustancias cuando se aplica la SURPS en adolescentes (Conrod, Castellanos-Ryan, y Mackie, 2011; Conrod, Castellanos-Ryan, y Strang, 2010; Mahu, Doucet, O'Leary-Barrett, y Conrod, 2015; O’Leary-Barrett, Castellanos-Ryan, Pihl, y Conrod, 2016) y en estudiantes universitarios (Kazemi, Levine, Dmochowski, Van Horn, y Qi, 2015).

La SURPS cuenta con algunas ventajas respecto a otros instrumentos que evalúan rasgos de personalidad. Primero, evalúa cuatro rasgos de personalidad de manera independiente, con un mínimo solapamiento de varianza (Woicik et al, 2009). Dicha característica permite a sus usuarios evitar la administración de escalas diferentes que no fueron diseñadas para evaluar estos constructos de forma independiente (Krank et al., 2011). Segundo, su brevedad permite que sea utilizada en grandes estudios epidemiológicos y longitudinales (Woicik et al., 2009). Tercero, permite identificar a personas en riesgo de consumo de sustancias, y sus problemas relacionados, sin preguntar sobre el uso de drogas, algo especialmente útil en la intervención preventiva con adolescentes (Castellanos-Ryan, O'Leary-Barrett, y Conrod, 2013).
La SURPS se desarrolló y validó inicialmente con muestras comunitarias, mayoritariamente adolescentes y estudiantes universitarios (Woicik et al., 2009). Desde entonces, numerosos estudios han mostrado sus adecuadas propiedades psicométricas para la interpretación de las puntuaciones propuestas. Estos estudios han sido llevados a cabo en diferentes subpoblaciones, principalmente adolescentes (Ali et al., 2016; Castellanos-Ryan et al., 2013; Castonguay-Jolin et al., 2013; Chandrika, Seneviratne, Newcombe, y Wanigaratne, 2009; Jurk et al., 2015; Krank et al., 2011; Malmberg et al., 2010; Memetovic, Ratner, Gotay, y Richardson, 2016; Newton et al., 2015; Robles-García et al., 2014; Siu, 2010; Woicik et al., 2009) pero también con muestras de estudiantes universitarios (Omiya, Kobori, Tomoto, Igarashi, y Iyo, 2015), adultos (Canfield, Gilvarry, y Koller, 2015; Saliba, Moran, y Yoo, 2014), personas encarceladas (Anthony y Brunelle, 2016) y pacientes hospitalizados (Schlauch, Crane, Houston, y Lang, 2015). Estos estudios han replicado la estructura de 4 factores de la SURPS (Krank et al., 2011; Saliba et al., 2014; Schlauch et al., 2015) y han mostrado evidencias de validez convergente y discriminante (Castellanos-Ryan et al., 2013; Newton et al., 2015; Robles-García et al., 2014).

La aplicabilidad de la SURPS se evidencia en el hecho de que ha sido traducida a diferentes idiomas como: sinhala (Chandrika et al., 2009), neerlandés (Malmberg et al., 2010), chino (Siu, 2010), coreano (Saliba et al., 2014), francés (Castonguay-Jolin et al., 2013; Jurk et al., 2015), alemán (Jurk et al., 2015), portugués (Canfield et al., 2015) y japonés (Omiya et al., 2015). La validación de la versión española de la SURPS (Robles-García et al., 2014) se realizó con 671 adolescentes mexicanos hispanohablantes (con edades entre 11-18 años). Esta traducción al español mostró una consistencia interna moderada y buenas evidencias de validez concurrente para las cuatro subescalas. Las subescalas IMP, SS, y H también predecían el futuro consumo de drogas, pero la subescala AS no mostró evidencias de validez predictiva. En cuanto a las evidencias de validez estructural, dicha versión española-mexicana de la SURPS replicó la estructura de cuatro factores de la versión original (Woicik et al., 2009), con la excepción del ítem 22 («Manipulo a los demás para conseguir lo que quiero») que tuvo una comunalidad menor de 0,40 en todos los factores y se excluyó del análisis. Esta carga factorial problemática del ítem 22 también se ha encontrado en la versión inglesa-canadiense con adolescentes jóvenes (Krank et al., 2011), la versión japonesa (Omiya et al., 2015), neerlandesa (Malmberg et al., 2010), inglesa-australiana y coreana (Saliba et al., 2014).

Para garantizar la equivalente en el proceso de adaptación de una medida a un idioma y población nuevos, se considera insuficiente la traducción directa e inversa. También es necesario realizar estudios teóricos sobre la equivalencia del constructo, así como considerar las particu- 
laridades lingüísticas y culturales de la población objetivo (Muñiz, Elosua, y Hambleton, 2013). Además, durante la validación de instrumentos, es necesario aportar evidencias sobre cómo pueden ser interpretadas las puntuaciones en las diferentes subpoblaciones y contextos en que el instrumento es aplicado (APA, AERA, NCME, 2014). Por ejemplo, las propiedades psicométricas de la versión francesa de la SURPS se han evaluado con muestras canadienses (Castonguay-Jolin et al., 2013) y francesas (Jurk et al., 2015). Por lo tanto, aunque se han realizado comparaciones interculturales o interlingüísticas de la SURPS (Canfield et al., 2015; Jurk et al., 2015), aún no se ha realizado un análisis de la fiabilidad y evidencias de validez de la versión española con una muestra reclutada en España.

Considerando las grandes diferencias culturales y lingüísticas entre España y México, el propósito de este estudio fue analizar las propiedades psicométricas de la nueva versión de la SURPS para estudiantes universitarios españoles. En concreto, pretendíamos ofrecer i) estimaciones de fiabilidad para la SURPS, ii) evidencias de su validez estructural, iii) evidencias de validez concurrente en base a sus asociaciones con otras medidas de personalidad, y iv) evidencias de la relación entre la SURPS y el consumo de alcohol. Tomando en consideración estudios previos, nuestras hipótesis son:

1. Considerando lo aportado por estudios anteriores sobre las propiedades psicométricas de la SURPS (Chandrika et al., 2009; Jurk et al., 2015; Malmberg et al., 2010; Robles-García et al., 2014; Saliba et al., 2014), nuestra hipótesis es que la estimación de fiabilidad mostrará valores entre moderados y buenos.

2. Se replicará la estructura de cuatro factores de la SURPS.

3. Las subescalas de la SURPS se asociarán con otras medidas del mismo constructo, específicamente: AS correlacionará con el Inventario de Ansiedad Estado-Rasgo (Spielberg, Gorsuch, y Lushene, 1970; Spielberg, Gorsuch, y Lushene, 2008); H correlacionará con la Escala de Desesperanza de Beck (Beck, Weissman, Lester, y Trxier, 1974); SS correlacionará con la subescala de búsqueda de sensaciones de la Escala de comportamiento impulsivo UPPSP (Lynam, Smith, Whiteside, y Cyders, 2006); e IMP correlacionará con las puntuaciones de todas las subescalas de la Escala de comportamiento impulsivo UPPSP (Lynam et al., 2006).

4. En estudios anteriores, Krank et al. (2011) detectaron que las subescalas IMP, SS y H eran los predictores más consistentes y fuertes del uso/abuso de drogas, mientras que Woicik et al. (2009) detectaron que estas tres subescalas estaban asociadas con la severidad de problemas relacionados con el alcohol en una muestra de estudiantes universitarios. Considerando lo anterior, mantenemos la hipótesis de que las subescalas IMP, SS y H estarán relacionadas con el uso problemático de alcohol medido mediante el test AUDIT (Prueba de Identificación de Trastornos Relacionados con el Consumo de Alcohol) (Saunders, Aasland, Babor, de la Fuente, y Grant, 1993).

\section{Método}

\section{Participantes}

La muestra de participantes del estudio estuvo formada por 455 estudiantes universitarios de cuatro universidades españolas: $62,2 \%$ de la Universidad de Huelva, $16 \%$ de la Universidad de Almería, 13,4\% de la Universidad de Cádiz y $8,4 \%$ de la Universidad de Granada. Se accedió a la muestra mediante un muestreo de conveniencia. El 78,9\% de la muestra fueron mujeres, y las edades estuvieron comprendidas entre los 18-55 años (media $=21,53$; DT $=5,146$ ) y no hubo diferencias estadísticamente significativas en edad según sexo.

En términos de consumo de sustancias, el 89,5\% de los participantes informó de haber consumido alcohol durante el mes previo a la administración del cuestionario. En cuanto al uso de cannabis, el 28,9\% lo consumió durante el año previo a la entrevista, y el $23 \%$ durante el último mes.

\section{Instrumentos}

La Substance Use Risk Profile Scale (SURPS) (Woicik et al., 2009) es una escala de 23 ítems agrupados en cuatro dimensiones: Desesperanza (ítems 1, 4, 7, 13, 17, 20, 23), sensibilidad a la ansiedad (ítems 8, 10, 14, 18, 21); impulsividad (ítems 2, 5, 11, 15, 22) y búsqueda de sensaciones (ítems 3, 6, 9, 12, 16, 19). El formato de respuesta se expresa en una escala de 4 puntos tipo Likert entre 1-4 ( $1=$ totalmente en desacuerdo, 2 = en desacuerdo, 3 = de acuerdo, 4 = totalmente de acuerdo) que se suman para obtener la puntuación total de cada subescala (ítems 1, 4, 7, 13, 20 y 23 tienen puntuación inversa).

La SURPS fue adaptada lingüísticamente al contexto español por parte del equipo de investigación con una muestra de estudiantes universitarios. Para esta adaptación, se siguieron las directrices para el control de calidad en la adaptación de pruebas propuestas por Muñiz, Elosua, y Hambleton (2013) y las directrices para la adaptación de pruebas propuestas por la International Test Commission (2010) (http://www.intestcom.org). Un panel de tres expertos analizó la equivalencia de los cuatro constructos evaluados, pero determinados aspectos culturales reflejados en la redacción de los ítems, así como aspectos gramaticales y relacionados con la redacción de la mayoría de ellos, no se consideraron apropiados para el contexto español.

Dos de los expertos que participaron en la adaptación tenían formación psicométrica y eran expertos en la investigación sobre trastornos por consumo de drogas. El otro 
experto tenía experiencia amplia profesional y de investigación en el campo del consumo recreativo de drogas. Estos expertos, considerando la versión original (Woicik et al., 2009) y la versión española-mexicana (Robles-García et al., 2014) de la SURPS, adaptaron, de forma independiente, los ítems a los aspectos culturales de la población española.

Inicialmente, cada experto tradujo la versión en inglés al castellano. Después, se analizó la concordancia de los ítems de las tres traducciones. Los ítems de los tres expertos que eran iguales fueron directamente insertados en la versión adaptada. Se hizo un análisis contextual de las diferencias entre las traducciones de los expertos (p. ej., «Me gustaría tirarme en paracaídas»). Para estos ítems, los expertos tomaron una decisión conjunta respecto de un contexto equivalente para la población española y eligieron la versión definitiva del ítem. Después, el equipo de investigación evaluó la equivalencia del ítem propuesto. En este sentido, el estudio sigue las directrices propuestas por algunos autores (Carretero-Dios y Pérez, 2007) y por normas internacionales (International Test Commission, 2010).

La Impulsive Behavior Scale, UPPSP (Lynam et al., 2006) es un inventario de 59 ítems que mide cinco dimensiones de personalidad específicas del comportamiento impulsivo: (falta de) premeditación, (falta de) perseverancia, urgencia negativa, búsqueda de sensaciones, y urgencia positiva. La versión original del UPPS estaba compuesta por cuatro dimensiones (Whiteside y Lynam, 2001); sin embargo, a partir de los estudios de Smith y sus colegas, se incluyó una quinta dimensión (Cyders et al., 2007). Los ítems del UPPS-P se puntúan en una escala de 4 puntos (de «totalmente de acuerdo» a «totalmente en desacuerdo»). Se utilizó la versión española desarrollada por Verdejo-García, Lozano, Moya, Alcázar, y Pérez-García (2010).

Se administró la subescala de ansiedad estado del Inventario de Ansiedad Estado-Rasgo (Spielberg et al., 1970; Spielberg et al., 2008). Esta subescala se compone de 20 ítems en una escala tipo Likert de cuatro puntos, donde una mayor puntuación es indicativa de una mayor ansiedad.

Escala de Desesperanza de Beck (Beck et al., 1974). Se utilizó la versión española de Aguilar et al. (1995). Esta escala está compuesta de 20 ítems con respuestas en formato verdadero-falso. La puntuación máxima, indicativa de mayor desesperanza, es 20, y la mínima 0.

Test de Identificación de los Trastornos Debidos al Consumo de Alcohol (AUDIT) (Saunders et al., 1993). El AUDIT es un instrumento de cribado compuesto de 10 ítems desarrollados por la Organización Mundial de la Salud para identificar consumo de alcohol de riesgo y perjudicial. Puntuaciones superiores a siete indican consumo problemático de alcohol, con valores de sensibilidad y especificidad mayores de 0,9 y 0,8, respectivamente (Babor, Biddle-Higgins, Saunders, y Monteiro, 2001).

\section{Procedimiento}

El test se administró a grupos de entre 38-45 estudiantes. A los sujetos que participarían en el retest $(\mathrm{N}=60)$ se les asignó un código para garantizar el anonimato. El intervalo de tiempo entre las administraciones test-retest fue una semana. Los participantes fueron informados de la duración del cuestionario (aproximadamente $20 \mathrm{mi}$ nutos) y de su anonimato. Todos los participantes dieron su consentimiento para participar de forma voluntaria en el estudio. El Comité de Bioética en estudios humanos de la Universidad de Huelva aprobó el protocolo del estudio.

\section{Análisis de datos}

Se eliminaron de los análisis siete participantes (1,5\%) que no habían respondido a algún ítem de la SURPS. Para comprobar la normalidad de las puntuaciones de la SURPS, se calcularon los valores de asimetría y curtosis, obteniendo todos los ítems valores aceptables (asimetría, $-0,76,-1,35$; curtosis, $-1,37,1,24)$.

La fiabilidad de las subescalas de la SURPS se estimó con el coeficiente de consistencia interna alfa de Cronbach, el coeficiente omega (McDonald, 1999), el coeficiente de dos mitades y el coeficiente test-retest. Se obtuvieron evidencias de validez convergente de las subescalas de la SURPS mediante el análisis de su asociación (coeficientes de correlación de Pearson) con las puntuaciones totales en la Escala de Desesperanza de Beck, el Inventario de Ansiedad Estado (STAI) y la Escala de comportamiento impulsivo UPPSP. Las puntuaciones compuestas se calcularon para cada subescala de la SURPS. Se obtuvieron las correlaciones bivariadas entre las diferentes escalas de la SURPS y el resto de medidas teóricamente relevantes administradas como parte de este estudio.

Los participantes fueron clasificados en dos grupos (consumo problemático vs. no problemático de alcohol). Para ello se consideró un punto de corte de 8 en las puntuaciones totales del AUDIT (Babor et al., 2001). Se implementaron pruebas t independientes para analizar las diferencias entre los grupos en cada subescala de la SURPS. Se calcularon las correlaciones de spearman entre las subescalas de la SURPS y los ítems 1 a 8 del AUDIT.

Finalmente, se comprobó la estructura factorial de la SURPS mediante un Análisis Factorial Confirmatorio (AFC) usando una matriz de varianza-covarianza. Se consideraron criterios tradicionales como indicativos de adecuado ajuste (CFI, NNFI $>0,90$ y RMSEA $<0,08$ ). Las interpretaciones de las cargas factoriales sugieren que una carga mayor de 0,71 debería considerarse excelente, 0,63 muy buena, 0,55 buena, 0,45 adecuada y 0,32 pobre. El método de los multiplicadores de Lagrange (prueba de ML) y la prueba Wald fueron utilizadas como índices de modificación para cada modelo. Se sometieron a prueba dos modelos: El Modelo 1 probó la estructura de cuatro factores, 
con todos los factores correlacionados. Como alternativa, el Modelo 2 (también sometido a prueba por Woicik et al., 2009) incluyó dos factores de segundo orden correlacionados: las dimensiones de personalidad de neurosis (incluyendo ítems en los factores $\mathrm{H}$ y AS) y de desinhibición (con ítems asociados a los factores SS e IMP).

Antes de realizar el AFC con los 22 ítems de la escala (excluyendo el ítem 22), se detectó falta de normalidad multivariante (coeficiente estándar de Mardia = 40,840), por lo que se utilizó el método de Máxima Verosimilitud con errores estándares robustos.

Para el análisis de datos se utilizaron los paquetes estadísticos SPSS 21.0 (IBM SPSS Statistics, 2012) y EQS 6.2 (Bentler, 1995).

\section{Resultados}

\section{Análisis de ítems y fiabilidad}

La Tabla 1 muestra las puntuaciones medias, las desviaciones típicas y los índices de discriminación en cada subescala. Las puntuaciones medias más bajas se observaron en la subescala de Desesperanza (puntuaciones medias entre 1,36-1,90). Para la mayoría de los ítems en las demás subescalas se obtuvieron puntuaciones cercanas a sus valores medios. Todos los ítems mostraron valores aceptables de asimetría y de curtosis. Los índices de discriminación de todos los ítems fueron aceptables (correlaciones ítem-total corregidas entre 0,30-0,62) con la excepción de ítem 22 («Manipulo a los demás para conseguir lo que quiero») de la subescala de impulsividad $(r=0,13)$. Este funcionamiento problemático del ítem 22 también se ha encontrado en otros estudios psicométricos previos de la SURPS. Por ello, el ítem fue omitido del resto de análisis. Las correlaciones ítem-total de cada ítem con las demás subescalas mostraron valores más bajos a los mostrados con sus propias subescalas.

Los valores de Cronbach para la estimación de consistencia interna variaron entre $0,652-0,806$, mientras que los valores de los coeficientes omega variaron entre 0,654 0,807 . La fiabilidad estimada mediante los coeficientes de dos mitades varió entre 0,686-0,829. La estimación de fiabilidad test-retest varió entre 0,733-0,868.

La fiabilidad de los demás instrumentos administrados en nuestro estudio se estimó mediante el coeficiente de consistencia interna alfa de Cronbach y el coeficiente de dos mitades. Los resultados fueron:

UPPS-P: Los coeficientes alfa de consistencia interna fueron: (falta de) premeditación, 0,808: urgencia negativa, 0,841: (falta de) perseverancia, 0,770: búsqueda de sensaciones, 0,863: urgencia positiva, 0,923. Los coeficientes de dos mitades fueron: (falta de) premeditación, 0,859: urgencia negativa, 0,780: (falta de) perseverancia, 0,716: búsqueda de sensaciones, 0,863: urgencia positiva, 0,874 .
Tabla 1. Análisis de los ítems de la SURPS

\begin{tabular}{|c|c|c|c|c|c|}
\hline & $M$ & DT & Asimetría & Curtosis & $\begin{array}{l}\text { Correlación } \\
\text { ítem-total }\end{array}$ \\
\hline \multicolumn{6}{|l|}{ Desesperanza } \\
\hline Ítem 1 (I) & 1,90 & 0,54 & 0,11 & 0,99 & 0,47 \\
\hline Ítem 4 (I) & 1,75 & 0,62 & 0,38 & 0,19 & 0,59 \\
\hline Ítem 7 (I) & 1,76 & 0,61 & 0,37 & 0,22 & 0,50 \\
\hline Ítem 13 (I) & 1,51 & 0,61 & 1,00 & 1,09 & 0,50 \\
\hline Ítem 17 & 1,36 & 0,56 & 1,35 & 1,25 & 0,56 \\
\hline Ítem 20 (I) & 1,83 & 0,65 & 0,38 & 0,05 & 0,62 \\
\hline Ítem $23(I)$ & 1,66 & 0,64 & 0,66 & 0,33 & 0,52 \\
\hline Alfa de Cronbach & \multicolumn{5}{|c|}{0,806} \\
\hline Coeficiente Omega & \multicolumn{5}{|c|}{0,807} \\
\hline Fiabilidad de dos mitades & \multicolumn{5}{|c|}{0,829} \\
\hline Fiabilidad test-retest & \multicolumn{5}{|c|}{0,755} \\
\hline \multicolumn{6}{|l|}{ Sensibilidad a la ansiedad } \\
\hline Ítem 8 & 2,24 & 0,87 & 0,24 & $-0,65$ & 0,44 \\
\hline Ítem 10 & 2,19 & 0,80 & 0,25 & $-0,42$ & 0,40 \\
\hline Ítem 14 & 2,32 & 0,76 & 0,15 & $-0,33$ & 0,42 \\
\hline Ítem 18 & 2,30 & 0,73 & $-0,06$ & $-0,47$ & 0,47 \\
\hline Ítem 21 & 2,18 & 0,78 & 0,29 & $-0,29$ & 0,30 \\
\hline Alfa de Cronbach & \multicolumn{5}{|c|}{0,652} \\
\hline Coeficiente Omega & \multicolumn{5}{|c|}{0,654} \\
\hline Fiabilidad de dos mitades & \multicolumn{5}{|c|}{0,686} \\
\hline Fiabilidad test-retest & \multicolumn{5}{|c|}{0,793} \\
\hline \multicolumn{6}{|l|}{ Impulsividad } \\
\hline Ítem 2 & 2,00 & 0,71 & 0,49 & 0,35 & 0,48 \\
\hline Ítem 5 & 2,55 & 0,75 & $-0,20$ & $-0,29$ & 0,32 \\
\hline Ítem 11 & 1,97 & 0,69 & 0,42 & 0,26 & 0,59 \\
\hline Ítem 15 & 2,39 & 0,84 & 0,12 & $-0,56$ & 0,43 \\
\hline Ítem 22 & 1,65 & 0,73 & 0,82 & $-0,11$ & 0,13 \\
\hline Alfa de Cronbach ${ }^{1}$ & \multicolumn{5}{|c|}{0,685} \\
\hline Coeficiente Omega & \multicolumn{5}{|c|}{0,654} \\
\hline Fiabilidad de dos mitades ${ }^{1}$ & \multicolumn{5}{|c|}{0,710} \\
\hline Fiabilidad test-retest ${ }^{1}$ & \multicolumn{5}{|c|}{0,733} \\
\hline \multicolumn{6}{|l|}{ Búsqueda de sensaciones } \\
\hline Ítem 3 & 2,64 & 1,15 & $-0,25$ & $-1,38$ & 0,50 \\
\hline Ítem 6 & 3,39 & 0,64 & $-0,76$ & 0,20 & 0,39 \\
\hline Ítem 9 & 2,51 & 0,78 & $-0,22$ & $-0,40$ & 0,39 \\
\hline Ítem 12 & 2,22 & 1,08 & 0,29 & $-1,24$ & 0,39 \\
\hline Ítem 16 & 2,26 & 0,87 & 0,29 & $-0,59$ & 0,46 \\
\hline Ítem 19 & 2,83 & 0,90 & $-0,39$ & $-0,63$ & 0,54 \\
\hline Alfa de Cronbach & \multicolumn{5}{|c|}{0,706} \\
\hline Coeficiente Omega & \multicolumn{5}{|c|}{0,722} \\
\hline Fiabilidad de dos mitades & \multicolumn{5}{|c|}{0,716} \\
\hline Fiabilidad test-retest & \multicolumn{5}{|c|}{0,868} \\
\hline
\end{tabular}

Nota. (I) Puntuación inversa.

${ }^{1}$ El ítem 22 fue excluido del cálculo de este valor. 
Inventario de Ansiedad Estado-Rasgo: La consistencia interna estimada como Alfa de Cronbach fue 0,888 y el coeficiente de dos mitades 0,897 .

Escala de Desesperanza de Beck: La consistencia interna estimada como Alfa de Cronbach fue 0,717 y el coeficiente de dos mitades 0,724.

AUDIT: La consistencia interna estimada como Alfa de Cronbach fue 0,786 y el coeficiente de dos mitades 0,737.

\section{Evidencia de validez basadas en la estructura interna}

Considerando los valores de chi cuadrado y RMSEA, el modelo de cuatro factores no mostró un ajuste adecuado, pero no fue el caso de los valores CFI o NCFI $\left(\chi^{2}{ }_{203}=429,99\right.$; $\mathrm{CFI}=0,87$, NCFI $=0,856$; $\mathrm{RMSEA}=0,05[95 \% \mathrm{IC}=0,04$, $0,05]$.

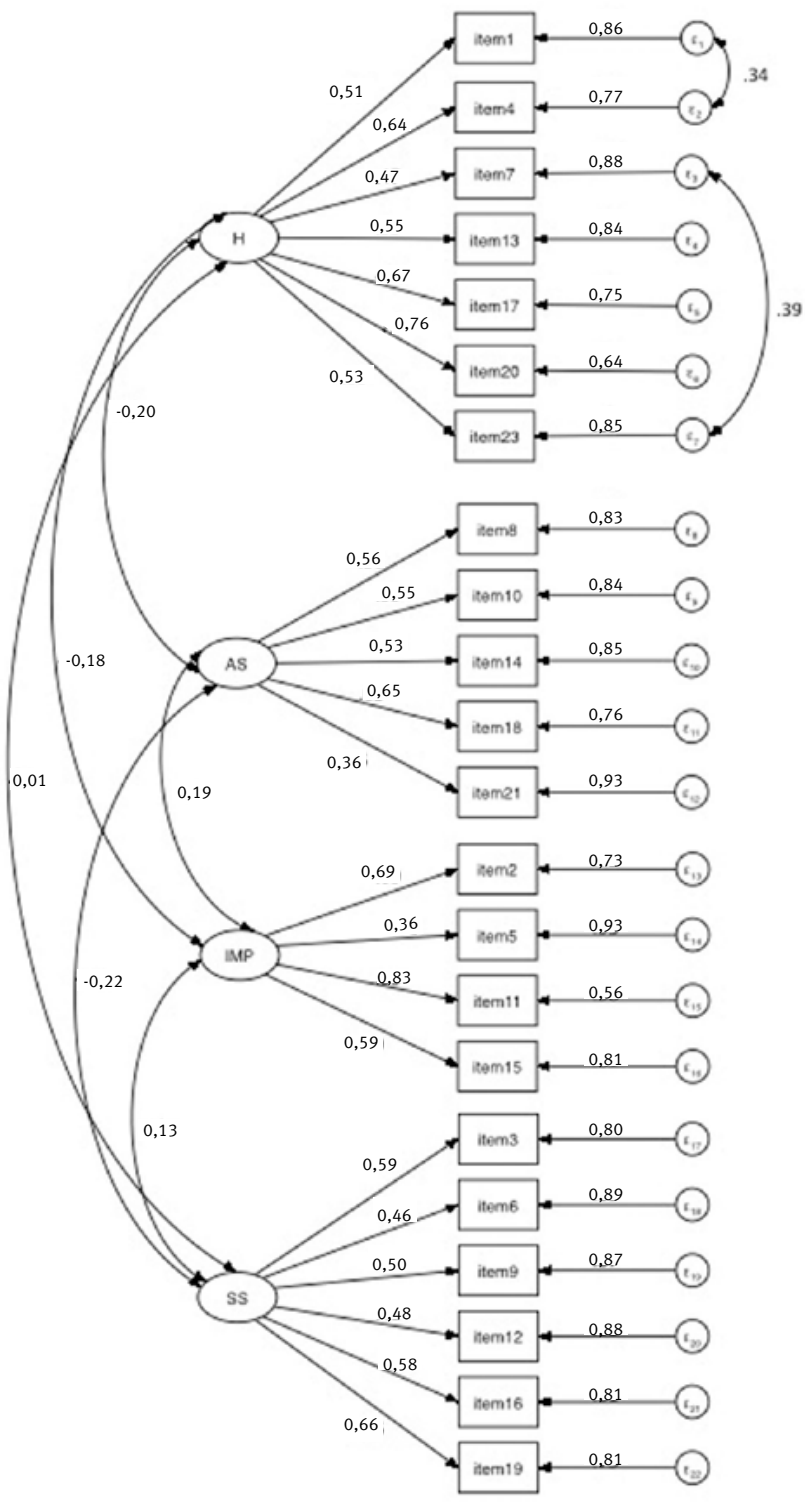

Figura 1. Modelo y cargas factoriales de la versión española de la SURPS
Los resultados de la prueba Wald sugirieron la inclusión de covarianza entre los términos de error de los ítems 23 y 7 , y entre los ítems 4 y 1 , por lo que las estimaciones de ambos parámetros fueron incluidos en un segundo modelo. Los errores correlacionados fueron atribuidos a la similitud en la redacción de ambos ítems. Dicho modelo modificado mostró un adecuado ajuste $\left(\chi_{203}^{2}=340,32 ; \mathrm{CFI}=0,92\right.$, $\mathrm{NCFI}=0,91 ;$ RMSEA $=0,04[95 \%$ IC $=0,03,0,04] \mathrm{y}$, como se esperaba, se replicó la estructura de cuatro factores de la SURPS. Las cargas factoriales estandarizadas de los ítems en cada factor variaron entre 0,359-0,831. Todas las cargas y correlaciones mayores a 0,13 fueron significativamente diferentes de cero con $p<.001$, excepto la correlación entre Desesperanza y Búsqueda de sensaciones. El modelo alternativo (modelo 2) con dos factores de segundo orden mostró menor ajuste $\left(\chi_{203}^{2}=373,32 ; \mathrm{CFI}=0,90\right.$, NCFI $=$ 0,$89 ;$ RMSEA $=0,04[95 \% \mathrm{IC}=0,03,0,05]$.

\section{Evidencias de validez convergente: Evidencias basa-} das en la relación con otras medidas de personalidad

Como muestra la Tabla 2, las puntuaciones en las diferentes subescalas de la SURPS mostraron relación con otras medidas del mismo constructo. La escala SURPS de Desesperanza correlacionó de forma moderada y significativa con la Escala de Desesperanza de Beck $(r=0,58, p$ $<.001)$ y con el STAI $(r=0,61, p<.001)$. Las puntuaciones de Sensibilidad a la ansiedad correlacionaron con el STAI $(r=0,40, p<.001)$. La escala de Impulsividad mostró relaciones significativas con todas las medidas de personalidad, a excepción de la Escala de Desesperanza de Beck, mostrando una correlación más fuerte con Falta de Premeditación $(r=0,52, p<.001)$ y Urgencia Negativa $(r=0,61, p$ $<.001)$. Por último, la escala de Búsqueda de Sensaciones

Tabla 2. Correlaciones entre la SURPS y otras medidas de personalidad

\begin{tabular}{|c|c|c|c|c|}
\hline & H & AS & IMP & SS \\
\hline $\begin{array}{l}\text { UPPS1. Falta de } \\
\text { premeditación }\end{array}$ & 0,01 & $-0,05$ & $0,52^{\star \star}$ & 0,20 ** \\
\hline UPPS2. Urgencia negativa & $0,29 * \star$ & $0,26^{\star \star}$ & $0,61^{\star \star}$ & $0,17^{\star}$ \\
\hline $\begin{array}{l}\text { UPPS3. Falta de } \\
\text { perseverancia }\end{array}$ & $0,35^{\star \star}$ & 0,06 & $0,22^{\star \star}$ & $0,11^{\star}$ \\
\hline $\begin{array}{l}\text { UPPS4. Búsqueda de } \\
\text { sensaciones }\end{array}$ & $-0,01$ & $-0,12^{\star}$ & $0,13^{\star}$ & $0,81^{* *}$ \\
\hline UPPS5. Urgencia positiva & $0,20 * \star$ & $-0,18^{\star \star}$ & $0,38^{\star \star}$ & $0,29 * \star$ \\
\hline Beck & $0,58^{\star \star}$ & $0,11^{\star \star}$ & 0,07 & $-0,01$ \\
\hline STAI & $0,61^{\star \star}$ & $0,40^{\star \star}$ & $0,29^{\star \star}$ & $-0,10$ \\
\hline
\end{tabular}

Nota. ${ }^{\star} p<, 05 ;{ }^{\star \star} p<, 01$ 
mostró una correlación fuerte con la escala de Búsqueda de Sensaciones de la UPPS-P $(r=0,82, p<.001)$ (Tabla 2$)$.

\section{Evidencias de validez basadas en la relación con otras variables: Relación con consumo de alcohol}

Las Tablas 3 y 4 muestran el análisis entre las subescalas de la SURPS y las puntuaciones en el AUDIT, concretamente, con su puntuación total (uso problemático), y

Tabla 3. Relación entre consumo de alcohol y puntuaciones en la SURPS

\begin{tabular}{lcccccc}
\hline & $\begin{array}{c}\text { Uso } \\
\text { problemático }\end{array}$ & $\mathbf{n}$ & Media (SD) & t & P & d \\
\hline Desesperanza & No & 348 & $11,67(2,88)$ & 1,84 &, 066 & 0,21 \\
& Sí & 100 & $12,27(2,92)$ & & & \\
Impulsividad & No & 348 & $10,26(2,33)$ & 5,46 & $<$ & 0,63 \\
& Sí & 100 & $11,69(2,23)$ & &, 001 & \\
Búsqueda de & No & 348 & $15,52(3,54)$ & 4,19 & $<$ & 0,49 \\
sensaciones & Sí & 100 & $17,18(3,15)$ & &, 001 & \\
Sensibilidad & No & 348 & $11,16(2,58)$ & 1,62 &, 107 & 0,18 \\
a la ansiedad & Sí & 100 & $11,63(2,51)$ & & & \\
\hline
\end{tabular}

Tabla 4. Correlaciones de spearman entre los ítems del AUDIT (1-8) y las puntuaciones en la SURPS entre quienes consumieron alcohol en el último año

\begin{tabular}{|c|c|c|c|c|}
\hline & Desesperanza & $\begin{array}{c}\text { Sensibilidad a la } \\
\text { ansiedad }\end{array}$ & Impulsividad & $\begin{array}{l}\text { Búsqueda de } \\
\text { sensaciones }\end{array}$ \\
\hline Ítem 1 & 0,045 & 0,039 & $0,186^{\star *}$ & $0,265^{\text {** }}$ \\
\hline Ítem 2 & 0,003 & 0,070 & $0,215^{\star \star}$ & $0,122^{\star \star}$ \\
\hline Ítem 3 & 0,067 & 0,052 & $0,234^{\star \star}$ & $0,245^{\star *}$ \\
\hline Ítem 4 & $0,150^{\star *}$ & 0,031 & $0,130^{\star \star}$ & $0,126^{\star \star}$ \\
\hline Ítem 5 & 0,075 & 0,075 & $0,179^{\star \star}$ & $0,159^{\star \star}$ \\
\hline Ítem 6 & $0,112^{\star}$ & 0,086 & $0,127^{\star}$ & 0,067 \\
\hline Ítem 7 & $0,167^{\star \star}$ & $0,204^{\star \star}$ & $0,187^{\star \star}$ & $0,197^{\star \star}$ \\
\hline Ítem 8 & $0,119^{\star}$ & 0,076 & $0,190^{\star \star}$ & $0,149^{\star \star}$ \\
\hline
\end{tabular}

Nota. ${ }^{\star} p<, 05 ;{ }^{* \star} p<, 01$

los ítems 1 a 8. Los ítems 9 y 10 fueron omitidos, debido al bajo número de participantes que informó de lesiones sufridas por otras personas debido a su ingesta de alcohol (ítem $9,6,4 \%$ ) o de haber preocupado a otra persona por su ingesta de alcohol (ítem 10,5,3\%).

Los participantes identificados como consumidores de alcohol problemáticos según el AUDIT mostraron puntuaciones más altas en las subescalas de impulsividad y búsqueda de sensaciones en comparación a los participantes sin uso de alcohol problemático (diferencia estadísticamente significativa) (Tabla 3). No se encontraron diferencias esta- dísticamente significativas entre las subescalas de ansiedad y desesperanza entre ambos grupos. Como se esperaba, se encontraron fuertes asociaciones entre el consumo de alcohol y las subescalas IMP y SS. Entre los participantes que consumieron alcohol durante el último mes, se encontraron correlaciones significativas entre todos los ítems y las puntuaciones en Impulsividad. La subescala de búsqueda de sensaciones correlacionó con todos los ítems, con la excepción del ítem 6 (Tabla 4).

\section{Discusión}

A pesar de la existencia de una versión española-mexicana de la SURPS (Robles-García et al., 2014), las grandes diferencias lingüísticas y culturales entre México y España justifican el desarrollo de una versión española evaluada en el contexto y la población de España. Este estudio analizó las propiedades psicométricas de una versión española revisada de la SURPS en una muestra de estudiantes universitarios reclutados en España. Los resultados mostraron que esta versión revisada tenía una fiabilidad buena y evidencias de validez basadas en su estructura interna y su asociación con otras medidas de personalidad y de consumo de sustancias.

Respecto a la fiabilidad, como expresamos en nuestra primera hipótesis, los resultados son coherentes con los obtenidos en otros estudios para la adaptación de la SURPS a otros idiomas, con valores de consistencia interna para las subescalas de la SURPS que variaron entre moderados a buenos (Chandrika et al., 2009; Jurk et al., 2015; Malmberg et al., 2010; Robles-García et al., 2014; Saliba et al., 2014). Entre los estudios de otras versiones traducidas de la SURPS, el estudio actual es uno de los pocos que analizó la fiabilidad test-retest, encontrando una fiabilidad test-retest buena en todas las escalas. En general, las correlaciones entre escalas halladas en nuestro estudio son más altas que las de otras versiones lingüísticas de la SURPS, como la japonesa (Omiya et al., 2015), singalesa (Chandrika et al., 2009), francesa o alemana (Jurk et al., 2015) y muy similares a las halladas respecto de la escala original (Woicik et al., 2009).

En coherencia con nuestra segunda hipótesis, nuestro estudio replicó la estructura de cuatro factores de la escala original (Woicik et al., 2009). Los dos errores de covarianza incluidos en el modelo sometido a prueba son coherentes con los resultados de Krank et al. (2011) y Woicik et al. (2009). Además, los resultados sugieren que se ha de eliminar el ítem 22 de la versión española de la SURPS, igual que sugirieron los resultados del estudio con las versiones española-mexicana (Robles-García et al., 2014), inglesa-canadiense con jóvenes adolescentes (Krank, 2011), japonesa (Omiya et al., 2015), neerlandesa (Malmberg et al., 2010), inglesa-australiana (Newton et al, 2015) y coreana de la SURPS (Saliba et al., 2014). 
Tercero, se aportaron evidencias de una buena validez convergente de las subescalas de la SURPS, mostrando una asociación significativa con otras escalas de personalidad relevantes en términos conceptuales. En línea con los resultados de Krank et al. (2011) y de Robles-García et al (2014), encontramos asociaciones significativas entre la subescala H y la Escala de Desesperanza de Beck. Además, nuestros resultados son similares a los hallados por Woicik et al. (2009), quienes indicaron: "La subescala IMP de la SURPS debería captar una dimensión de la personalidad diferente de la SS y que esté asociada con un uso de drogas más intenso y espontáneo" (pág. 1044). El hecho que la correlación entre la subescala IMP y la subescala SS del UPPS-P fuese solo leve, y que la correlación con la escala SS de la SURPS fuese muy fuerte, puede considerarse una evidencia discriminante que apoya las diferencias entre ambas dimensiones.

Anteriormente, otros investigadores han encontrado que las subescalas H, IMP y SS son los rasgos de personalidad de la SURPS más fuertemente asociadas con la frecuencia del uso de alcohol y con los problemas relacionados con el alcohol. Por ejemplo, Woicik et al. (2009) encontró que estas subescalas estaban asociadas con la severidad de problemas relacionados con el alcohol en una muestra de estudiantes universitarios, mientras que los resultados de Stewart et al (2011) mostraron que H, SS, e IMP tenían una correlación positiva con los problemas relacionados con el consumo de alcohol. En este estudio, IMP y SS correlacionaron con la frecuencia del uso de alcohol y con los problemas relacionados con el alcohol del AUDIT (con la excepción de SS y el ítem «¿Con qué frecuencia en el curso del último año ha necesitado beber en ayunas para recuperarse después de haber bebido mucho el día anterior?»). Estas dos subescalas tuvieron una asociación positiva con todas las medidas de alcohol analizadas por este estudio con estudiantes universitarios españoles, lo que debería tenerse en cuenta a la hora de diseñar e implementar intervenciones para minimizar o reducir los problemas relacionados con el consumo de alcohol.

Cabe destacar que la subescala AS no estuvo relacionada con el consumo de alcohol de riesgo, la frecuencia de su consumo o con episodios de atracón de alcohol; AS estaba correlacionada con el ítem «¿Con qué frecuencia en el curso del último año no ha podido recordar lo que sucedió la noche anterior porque habia estado bebiendo?». Como ya hemos mencionado, estudios anteriores también han encontrado que este rasgo de personalidad no se relaciona con estas medidas de consumo de alcohol. Es más, en algunos casos se ha hallado una relación negativa entre AS y frecuencia de uso de alcohol (Krank et al., 2011; Stewart et al., 2011; Woicik et al., 2009). La subescala H correlacionó con ítem 4 («¿Con qué frecuencia en el curso del último año ha sido incapaz de parar de beber una vez había empezado?»), ítem 6 («¿Con qué frecuencia en el curso del último año ha necesitado beber en ayunas para recuperarse después de haber bebido mucho el día anterior?»), ítem 7 («¿Con qué frecuencia en el curso del último año ha tenido remordimientos o sentimientos de culpa después de haber bebido?») e ítem 8 ( ¿¿Con qué frecuencia en el curso del último año no ha podido recordar lo que sucedió la noche anterior porque habia estado bebiendo?»). Se requiere una mayor investigación sobre el papel de estos rasgos de personalidad en el uso y abuso de alcohol. Nuestros resultados muestran que la frecuencia de consumo de alcohol y el atracón están relacionados con puntuaciones más altas en SS e IMP. Estas dos subescalas tuvieron una asociación positiva con todas las medidas de alcohol analizadas en este estudio con estudiantes universitarios españoles, lo que debería ser considerado para el diseño e implementación de intervenciones dirigidas a minimizar o reducir los problemas relacionados con el alcohol.

$\mathrm{Al}$ interpretar nuestros hallazgos, debemos considerar algunas limitaciones. Primero, la muestra no fue seleccionada de forma aleatoria de la población de estudiantes universitarios en España, lo que puede afectar la representatividad y la generalizabilidad de nuestros resultados. Esta limitación es común a muchos estudios psicométricos de la SURPS (con la excepción de Malmberg et al., 2010 y Castellanos et al., 2013). No obstante, con el fin de minimizar esta limitación, se seleccionaron estudiantes de cuatro universidades diferentes. Segundo, la mayoría de los participantes en este estudio eran mujeres, al igual que en otras versiones de la SURPS, como la japonesa (Omiya et al., 2015) y la portuguesa (Canfield et al., 2015). Esto puede influir en las propiedades psicométricas de la SURPS aportadas en nuestro estudio. No obstante, la invarianza de las cuatro dimensiones de la SURPS entre géneros ha sido ampliamente demostrada (Ali et al., 2016; Jurk et al., 2015; Memetovic, Ratner, Gotay, y Richardson, 2016; Woicik et al., 2009). Por último, dada la importancia de considerar las características de los participantes en el proceso de adaptación de la medida, sería conveniente ofrecer una descripción más detallada de las características sociodemográficas de los participantes.

A pesar de estas limitaciones, nuestro estudio aporta evidencias de las buenas propiedades psicométricas de esta versión española revisada de la SURPS. Además, aporta evidencias de su asociación con el consumo de alcohol según las expectativas teóricas. Por ello, esta versión española de la SURPS puede ser un instrumento útil para medir los rasgos de personalidad relacionados con la vulnerabilidad al uso y abuso de sustancias en el contexto de la investigación o en intervenciones preventivas enfocadas en la personalidad.

Futuros estudios deberían analizar sus propiedades psicométricas en otras subpoblaciones, particularmente en adolescentes, para los cuales el valor de esta escala en la prevención del abuso de sustancias es mayor. También, se requiere de estudios longitudinales para mostrar evidencias de validez predictiva de esta versión española revisada de la SURPS en el consumo de alcohol y otras drogas. 


\section{Conflicto de intereses}

Los autores declaran la inexistencia de conflicto de intereses.

\section{Agradecimientos}

Esta investigación ha sido financiada con el proyecto de investigación 'Análisis de la intervención de los pacientes con patología dual: estudio longitudinal sobre los factores que intervienen en la estabilidad psicopatológica y la calidad de vida (Referencia PI-0287-2014)' de la convocatoria Fundación Progreso y Salud.

\section{Referencias}

Aguilar, E. J., Hidalgo, M. D., Cano, R., López, J. C., Campillo, M. y Hernández Martí, M. (1995). Estudio prospectivo de la desesperanza en pacientes psicóticos: características psicométricas de la Escala de Desesperanza de Beck. Anales de Psiquiatría, 11, 121-125.

Ali, A., Carré, A., Hassler, C., Spilka, S., Vanier, A., Barry, C. y Berthoz, S. (2016). Risk factors for substances use and misuse among young people in France: what can we learn from the Substance Use Risk Profile Scale? Drug and Alcohol Dependence, 163, 84-91. doi:10.1016/j.drugalcdep.2016.03.027.

American Psychological Association, American Educational Research Association, y National Council on Measurement in Education (2014). Standards for educational $\mathcal{E}^{\circ}$ psychological tests. Washington, DC: American Psychological Association.

Anderson, K. G., Tapert, S. F., Moabad, I., Crowley, T. J. y Brown, S. A. (2007). Personality risk profile for conduct disorder and substance use disorders in youth. Addictive Behaviors, 32, 2377-2382.

Anthony, A. B. y Brunelle, C. (2016). Substance use in incarcerated male offenders: predictive validity of a personality typology of substance misusers. Addictive Behaviors, 53, 86-93. doi:10.1016/j.addbeh.2015.10.001.

Ávila Escribano, J. J., Sánchez Barba, M., Álvarez Pedrero, A., López Villarreal, A., Recio Pérez, J., Rodríguez Rodilla, M. y Fraile García, E. (2016). Predictive Capacity of Cloninger's temperament and caracter inventory (TCI-R) in alcohol use disorder outcomes. Adicciones, 28, 136-143. doi:10.20882/adicciones.711.

Babor, T.F., Biddle-Higgins, J.C., Saunders, J.B. y Monteiro, M.G. (2001). AUDIT: The Alcohol Use Disorders Identification Test: Guidelines for Use in Primary Health Care. Geneva, Switzerland: World Health Organization.

Beck, A., Weissman, A., Lester, D. y Trxier, L. (1974). The measurement of pessimism: the Hopelessness Scale. Journal of Consulting and Clinical Psychology, 42, 861-865. doi:10.1037/h0037562.
Bentler, P. M. (1995). EQS structural equations program manual. Multivariate Software.

Canfield, M., Gilvarry, C. y Koller, S. H. (2015). Psychometric properties of the Substance Use Risk Profile Scale Brazilian version. International Journal of Mental Health and Addictions, 13, 204-214. doi:10.1007/s11469-0149522-1.

Carretero-Dios, H. y Pérez, C. (2007). Standards for the development and the review of instrumental studies: considerations about test selection in psychological research. International Journal of Clinical and Health Psychology, 7, 863-882.

Castellanos-Ryan, N. y Conrod, P. J. (2012). Personality and substance misuse: Evidence for a four factor model of vulnerability. In: J. Verster, K. Brady, M. Galanter, P. J. Conrod. (Eds). Drug Abuse and Addiction in Medical Illness: Causes, Consequences and Treatment. (pp. 47-62.) New York, NY: Springer Press.

Castellanos-Ryan, N., O'Leary-Barrett, M. y Conrod, P. (2013). Substance use in childhood and adolescence: a brief overview of developmental processes and their clinical implications. Journal of Canadian Child and Adolescence Psychiatry, 22, 41-46.

Castonguay-Jolin, L., Pernier-Ménard, E., Castellanos-Ryan, N., Parents, S., Vitaro, F., Tremblay, R. E.,... Conrod, P. J. (2013). Validation de la traduction française de la SURPS pour une population d'adolescents québécois. Canadian Journal of Psychiatry, 58, 538-545.

Chandrika, C., Seneviratne, A., Newcombe, P., A. y Wanigaratne, S. (2009). A model of substance abuse risk: adapting to the Sri Lankan context. Evaluation Review, 33, 83-97. doi:10.1177/0193841X08325145.

Cho, S. B., Llaneza, D., C., Adkins, A. E., Cooke, M., Kendler, K., Clark, S. y Dick, D. (2015). Patterns of substance use across the first year of college and associated risk factors. Frontiers in Psychiatry, 6, 152. doi:10.3389/ fpsyt.2015.00152.

Conrod, P. J., Castellanos-Ryan, N. y Mackie, C. J. (2011). Long-term effects of a personality-targeted intervention to reduce alcohol use in adolescents. Journal of Consulting and Clinical Psychology, 79, 296-306. doi:10.1037/ a0022997.

Conrod, P., Castellanos-Ryan, N. y Strang, J. (2010). Brief, personality-targeted skills interventions and survival as a non-drug user over a 2 year period during adolescence. Archive General of Psychiatry, 67, 85-93. doi:10.1001/archgenpsychiatry.2009.173.

Cyders, M.A., Smith, G. T., Spillane, N. S., Fischer, S., Annus, A. M. y Peterson, C. (2007). Integration of impulsivity and positive mood to predict risky behavior: Development and validation of a measure of positive urgency. Psychological Assessment, 19, 107-118.

Gonzálvez, M.T., Espada, J.P., Guillén-Riquelme, A., Secades, R. y Orgilés, M. (2016). Association between per- 
sonality traits and substance use in Spanish adolescents. Adicciones, 28, 108-115. doi:10.20882/adicciones.777.

Hicks B. M., Durbin C. E., Blonigen D. M., Iacono W. G. y McGue M. (2012). Relationship between personality change and the onset and course of alcohol dependence in young adulthood. Addiction, 107, 540-548. doi:10.1111/j.1360-0443.2011.03617.x .

IBM SPSS Statistics. (2012). IBM SPSS Statistics 21.0 for Windows. Chicago: IBM.

International Test Commission (2010). Guidelines for Translating and Adapting Tests. Recuperado de http:// www.intestcom.org.

Jurk, S., Kuitunen-Paul, S., Kroemer, N. B., Artiges, E., Banaschewski, T., Bokde, A. L., ... IMAGEN consortium. (2015). Personality and substance Use: psychometric evaluation and validation of the Substance Use Risk Profile Scale (SURPS) in English, Irish, French, and German adolescents. Alcoholism, Clinical and Experimental Research, 39, 2234-2248. doi:10.1111/acer.12886.

Kazemi, D., Levine, M., Dmochowski, J., Van Horn, K. y Qi, L. (2015). Health behaviors of mandated and voluntary students in a motivational intervention program. Preventive Medicine Reports, 2, 423-428. doi:10.1016/j.pmedr.2015.05.004.

Krank, M., Stewart, S. H., O’Connor, R., Woicik, P. B., Wall, A. M. y Conrod, P. J. (2011). Structural, concurrent, and predictive validity of the Substance Use Risk Profile Scale in early adolescence. Addictive Behaviors, 36, 37-46. doi:10.1016/j.addbeh.2010.08.010.

Kotov, R., Gamez, W., Schmidt, F. y Watson, D. (2010). Linking 'big' personality traits to anxiety, depressive, and substance use disorders: a meta-analysis. Psychological Bulletin, 136, 768-821. doi:10.1037/a0020327.

Lynam, D. R., Smith, G. T., Whiteside, S. P. y Cyders, M. A. (2006). The UPPS-P: Assessing five personality pathways to impulsive behavior. West Lafayette, IN: Purdue University.

McDonald, R. P. (1999). Test theory: A unified treatment. Mahwah, NJ: Lawrence Erlbaum Associates. doi:10.1111/j.2044-8317.1981.tb00621.x

Mahu, I., Doucet, C., O'Leary-Barrett, M. y Conrod, P. J. (2015). Can cannabis use be prevented by targeting personality risk in schools? Twenty-four-month outcome of the adventure trial on cannabis use: a cluster-randomized controlled trial. Addiction, 110, 1625-1633. doi:10.1111/add.12991.

Malmberg, M., Overbeek, G., Monshouwer, K., Lammers, J., Vollebergh, W. y Engels, R. (2010). Substance use risk profiles and associations with early substance use in adolescence. Journal of Behavioral Medicine, 33, 474-485. doi:10.1007/s10865-010-9278-4.

Memetovic, J., Ratner, P. A., Gotay, C. y Richardson, C. G. (2016). Examining the relationship between personality and affect-related attributes and adolescents' intentions to try smoking using the Substance Use Risk Profile
Scale. Addictive Behaviors, 56, 36-40. doi:10.1016/j.addbeh.2016.01.002.

Mitchell, M. R. y Potenza, M. N. (2014). Addictions and personality traits: impulsivity and related constructs. $\mathrm{Cu}$ rrent Behavioral Neuroscience Reports, 1, 1-12. doi:10.1007/ s40473-013-0001-y.

Muñiz, J., Elosua, P. y Hambleton, R. K. (2013). Directrices para la traducción y adaptación de los tests: segunda edición. Psicothema, 25, 151-157.

Nees, F., Tzschoppe, J., Patrick, C., Vollstäd-Klein, S., Steiner, S., Poutska, L., ... IMAGEN Consortium (2012). Determinants of early alcohol use in healthy adolescents: the differential contribution of neuroimaging and psychological factors. Neuropsychopharmacology, 37, 986-995. doi:10.1038/npp.2011.282.

Newton, N. C., Barret, E. L., Castellanos-Ryan, N., Kelly, E., Champion, K. E., Stapinski, L., ... Teesson, M. (2015). The validity of the Substance Use Risk Profile Scale (SURPS) among Australian adolescents. Addictive Behaviors, 53, 23-30. doi:10.1016/j.addbeh.2015.09.015.

O'Leary-Barrett, M., Castellanos-Ryan, N., Pihl, R. O. y Conrod, P. J. (2016). Mechanisms of personality-targeted intervention effects on adolescent alcohol misuse, internalizing and externalizing symptoms. Journal of Consulting and Clinical Psychology, 84, 438-452. doi:10.1037/ ccp0000082.

Omiya, S., Kobori, O., Tomoto, A., Igarashi, Y. y Iyo, M. (2015). Personality and substance use in Japanese adolescent: the Japanese version of Substance Use Risk Profile Scale. Personality and Individual Differences, 76, 153157. doi:10.1016/j.paid.2014.11.034.

Robles-García, R., Fresán, A., Castellanos-Ryan, N., Conrod, P., Gómez, D., de Quevedo, ... Medina-Mora, M. E. (2014). Spanish version of the substance use risk profile scale: factor structure, reliability, and validity in Mexican adolescents. Psychiatry Research, 220, 113-117. doi:10.1016/j.psychres.2014.08.057.

Saliba, A. J., Moran, C. y Yoo, Y. (2014). The Substance Use Risk Profile Scale: comparison of norms and outcomes for Australian and Korean adults. International Journal of Mental Health and Addictions, 12, 538-547. doi:10.1007/ s11469-014-9490-5.

Saunders, J. B., Aasland, O. G., Babor, T. F., de la Fuente, J. R. y Grant, M. (1993). Development of the Alcohol Use Disorders Identification Test (AUDIT): WHO Collaborative Project on early detection of persons with harmful alcohol consumption. Addiction, 88, 791-804.

Schlauch, R., Crane, C., Houston, R. y Lang, A. (2015). Psychometric evaluation of the Substance Use Risk Profile Scale (SURPS) in an inpatient sample of substance users using cue-reactivity methodology. Journal of Psychopathology and Behavioral Assessment, 37, 231-246. doi:10.1007/ s10862-014-9462-x. 
Sher, K. J., Bartholow, B. D. y Wood, M. D. (2000). Personality and substance use disorders: a prospective study. Journal of Consulting and Clinical Psychology, 68, 363-371.

Siu, A. F. Y. (2010). Validation of the substance use risk profile scale for adolescents in Hong Kong. Journal of Psychoeducational Assessment, 29, 75-83. doi:10.1177/0734282910362044.

Spielberger, C. D., Gorsuch, R. L. y Lushene, R. E. (1970). Manual for the state-trait anxiety inventory. Palo Alto, C. A.: Consulting Psychologist's Press.

Spielberger, C. D., Gorsuch, R. L. y Lushene, R. E. (2008). STAI. Cuestionario de ansiedad estado-rasgo (7a ed. rev.). Madrid: TEA.

Stewart, S., McGonnell, M., Wekerle, C., Adlaf, E. y The MAP Longitudinal Study Research Team. (2011). Associations of personality with alcohol use behaviour receiving child welfare services. International Journal of Mental Health and Addiction, 9, 492-506.

Verdejo-García, A., Lozano, O. M., Moya, M., Alcázar, M. A. y Perez-García, M. (2010). Psychometric properties of a Spanish version of the UPPS-P Impulsive Behavior Scale: Reliability, validity and association with trait and cognitive impulsivity Journal of Personality Assessment, 92, 70-77. doi:10.1080/00223890903382369.

Whiteside, S. P. y Lynam, D. R. (2001). The Five Factor Model and impulsivity: using a structural model of personality to understand impulsivity. Personality and Individual Differences, 30, 669-689. doi:10.1016/S01918869(00)00064-7.

Woicik, P. A., Stewart, S. H., Pihl, R. O. y Conrod, P. J. (2009). The substance use risk profile scale: A scale measuring traits linked to reinforcement-specific substance use profiles. Addictive Behaviors, 34, 1042-1055. doi:10.1016/j.addbeh.2009.07.001. 